\title{
Correspondence
}

\section{Assessment of gestational age in twins}

Sir,

We read with interest the paper by Woods and Malan on the assessment of gestational age in twins (Archives, 1977, 52, 735). They found that the concordant twins gave a similar score for gestational age, whereas the discordant twins, with more than $15 \%$ difference in weight, showed a significant difference in the scores between the individual twins. However, although the score is significantly different, it is noteworthy that if the combined score of superficial and neurological criteria is used the actual difference in terms of gestational age between the two groups is only 0.7 weeks, which is in fact less than the standard deviation of the method itself (approximately 1 week). This suggests that the difference in actual gestational age between the individual discordant twins, although of interest and statistically significant, is for practical purposes unimportant.

Their findings also show that the score for superficial criteria is more influenced by variation in weight than the neurological score or total score and by itself would thus underscore gestation in small-for-dates infants. This reinforces the value of using a combination of superficial and neurological criteria in the assessment of gestation rather than trying to restrict it to a group of superficial criteria only.

LiLly M. S. Dubowitz and Victor Dubowitz

Department of Paediatrics and Neonatal Medicine, Institute of Child Health, Hammersmith Hospital, London, W.12.

Drs Woods and Malan comment:

We appreciate the interest Professor and Dr Dubowitz have shown in our paper. While the mean gestational age score differed between discordant twins by only 0.7 weeks, the smaller twin underscored by more than a week in 10 of the 29 pairs.

We wondered whether the same pattern of underscoring might be found in singletons born to Coloured mothers in Cape Town. These infants tend to be growthretarded and of a shorter gestation than UK standards (Jaroszewicz et al., 1975). 100 randomly selected singletons born to Coloured primipara were studied using the Dubowitz scoring method (Dubowitz et al., 1970). The mean $( \pm S D)$ total score was $53 \cdot 7 \pm 7 \cdot 3$ (38.8 weeks).
Neurological and external features scores were $27 \cdot 9 \pm 3 \cdot 7$ and $25 \cdot 7 \pm 4 \cdot 2$ respectively.

Is the relatively low external features score a consistent finding in other centres? If not, it may partially explain the shorter assessed gestation in the study population.

\section{L. Woods and A. F. MAlaN Department of Paediatrics and Child Health, Institute of Child Health, Red Cross War Memorial Hospital, Rondebosch, Cape 7700, South Africa.}

\section{References}

Dubowitz, L. M. S., Dubowitz, V., and Goldberg, C. (1970). Clinical assessment of gestational age in the newborn infant. Journal of Pediatrics, 77, 1-10.

Jaroszewicz, A. M., Schumann, D. E. W., and Keet, M. P. (1975). Intrauteriene groeistandaarde van Kaapse Kleurlingbabas. South African Medical Journal, 49, 568-572.

Sir,

May I comment on the paper by Woods and Malan. Their point is well made about using caution in estimating gestational age by a scoring system in growth-retarded infants. It is necessary, though, to divide twins so studied into further groups; namely, dizygous (DZ) and monozygous (MZ) twins; and the latter into those with dichorionic (DC) or with monochorionic (MC) placentas. All DZ twins have DC placentas, and those MZ twins that also do are less discordant for birthweight (and length) than are MZ-MC twins. This is not surprising since MZ-MC twins may have an unequal sharing of placental mass supplying each twin, and vascular anastomoses are present across the vascular equator that divides these two placenta parts. Thus, transfusion syndromes of varying degrees can also occur.

Although the above complicates the picture, it enhances the ability to study intrauterine growth retardation in one infant of a pair that are (usually) phenotypically identical. The key factor is that in all twins each twin has the same gestational age as the other. And in MZ-MC pairs, one twin of the pair may well exhibit all the features and growth patterns of a small-for-dates infant, and his twin those of a term one (Falkner, 1966, 1978). Perhaps for this area of study, assessment of gestational age by scoring methods is not too appropriate.

Frank FALKNER Fels Research Institute, Wright State University, Yellow Springs, Ohio 45387, USA. 